\title{
Clinical utility gene card for: Leri-Weill dyschondrosteosis (LWD) and Langer mesomelic dysplasia (LMD)
}

\author{
Juliette Albuisson $^{1,2}$, Sébastien Schmitt ${ }^{\star, 1}$, Sabine Baron ${ }^{3}$, Stéphane Bézieau ${ }^{1,2}$, Sara Benito-Sanz ${ }^{4,5}$ and \\ Karen E Heath ${ }^{4,5}$
}

European Journal of Human Genetics (2012) 20, doi:10.1038/ejhg.2012.64; published online 18 April 2012

\section{DISEASE CHARACTERISTICS}

1.1 Name of the disease (synonyms)

Leri-Weill dyschondrosteosis (dyschondrosteosis, Madelung deformity, included) (LWD), Langer mesomelic dysplasia (LMD), and idiopathic short stature (ISS).

\subsection{OMIM\# of the disease}

127300, 249700, 300582.

1.3 Name of the analysed genes or DNA/chromosome segments Short Stature Homeobox gene; SHOX (Xp22.33/Yp11.32) plus upstream and downstream SHOX regulatory regions localised in the pseudoautosomal region 1 (PAR1).

\subsection{OMIM\# of the gene(s)}

312865-400020.

\subsection{Mutational spectrum}

In $\sim 70 \%$ of cases, LWD is caused by haploinsufficiency because of heterozygous deletions of SHOX and its enhancer regions or intragenic point mutations. LMD is caused by homozygous or compound heterozygous mutations in SHOX or its enhancer regions. SHOX haploinsufficiency is also the primary cause of short stature in $\sim 2-5 \%$ of ISS patients. ${ }^{1-3}$

Deletions including SHOX or its downstream enhancers account for $\sim 90 \%$ of identified mutations. The first common PAR1 deletion located downstream of SHOX and encompassing a novel SHOX enhancer, has been identified in 30 LWD and ISS patients. ${ }^{1}$ Recently, the first deletion encompassing the upstream SHOX enhancers was reported in an ISS patient. ${ }^{4,5}$ List of large rearrangements in the PAR1 region are included in the Decipher v5.1 interactive web-based database (http://decipher.sanger.ac.uk), which incorporates a suite of tools designed to aid the interpretation of submicroscopic chromosomal imbalance notably for pathogenicity. The remaining $10 \%$ of mutations are missense and nonsense mutations account, the majority of which are located within exons 3 and 4, encoding the functionally important homeodomain in particular for DNA binding. ${ }^{6}$ SHOX mutations have been collated in the SHOX mutation database (www.shox.uni-hd.de). ${ }^{7}$ Of note, the p.A170P is the first founder SHOX mutation in the Gypsy LWD population, thus permitting this mutation to be the first screening step in this specific population. ${ }^{8}$ Partial or complete SHOX duplications have also been reported in LWD and ISS. ${ }^{9}$ The molecular defect remains unknown in the remaining $\sim 30 \%$ of LWD cases.

\subsection{Analytical methods}

Multiplex ligation-dependant probe amplification (MLPA) with SALSA kit P018 (MRC-Holland, Amsterdam, the Netherlands) can be applied to detect PAR1 deletions and duplications encompassing SHOX and the downstream enhancer region. Alternative methods such as quantitative PCR, microsatellite marker analysis, and fluorescence in situ hybridisation were previously undertaken.

High resolution melting analysis and/or bi-directional DNA sequencing of exons 2-6a and their flanking intronic sequences are employed for detection of point mutations and small insertions and deletions. Sequencing is recommended when LMD is suspected.

\subsection{Analytical validation}

MLPA validation: deletions and duplications encompassing a single probe should always be confirmed by alternative methods. Initially, sequencing should be undertaken to exclude the presence of a mutation or polymorphism in the probe binding sequence, resulting in a false positive result. If no variant is observed, qPCR or high density array CGH should be undertaken to confirm the presence of the deletion.

Sequencing validation: confirmation of mutation in an independent biological sample of the index case. Special care is required in the interpretation of variants of unknown clinical significance. The gold standard is functional analysis but this is usually not possible in a diagnostic set up. They should therefore be interrogated using conservation analysis, segregation analysis in the relatives of the index patient, analysis of at least 300 chromosomes from normal ethnically matched controls, and, if possible, predictive protein analysis. Thus, a statement must indicate the analysis that has been undertaken.

Recently, external quality assurance scheme has been established for genetic investigation of SHOX deficiency, details of this pilot scheme are available from European Molecular Genetics Quality Network (EMQN) web site (www.emqn.org). 
1.8 Estimated frequency of the disease

(incidence at birth ('birth prevalence') or population prevalence)

The population prevalence for LWD is $\sim 1$ in 1000 individuals in the European population. The prevalence of LMD is unknown, but less than 70 cases have been reported.

\subsection{If applicable, prevalence in the ethnic group of investigated} person

LMD has a higher prevalence in populations with a high incidence of consanguinity.

\subsection{Diagnostic setting}

\begin{tabular}{lll}
\hline & Yes & No \\
\hline A. (Differential) diagnostics & $\bigotimes$ & $\square$ \\
B. Predictive testing & $\bigotimes$ & $\square$ \\
C. Risk assessment in relatives & $\bigotimes$ & $\square$ \\
D. Prenatal & $\bigotimes$ & $\square$ \\
\hline
\end{tabular}

Comment: LWD is characterised by short stature, mesomelic shortening of the limbs, and characteristic bilateral abnormality of the wrists known as Madelung deformity. However, the phenotype is highly variable and includes presentations without clinical signs of Madelung deformity and/or normal height. If an alternative diagnosis is excluded, SHOX mutation screening could be performed. Prenatal diagnosis is technically feasible but it is only recommended for LMD.

\section{TEST CHARACTERISTICS}

\begin{tabular}{|c|c|c|c|c|}
\hline & \multicolumn{2}{|c|}{ Genotype or disease } & \multirow{2}{*}{$\begin{array}{l}\text { A: True positives } \\
\text { B: False positives }\end{array}$} & \multirow{2}{*}{$\begin{array}{l}\text { C: False negative } \\
\text { D: True negative }\end{array}$} \\
\hline & Present & Absent & & \\
\hline \multicolumn{5}{|l|}{ Test } \\
\hline \multirow[t]{2}{*}{ Positive } & $A$ & B & Sensitivity: & $A /(A+C)$ \\
\hline & & & Specificity: & $D /(D+B)$ \\
\hline \multirow[t]{2}{*}{ Negative } & C & D & Positive predictive value: & $A /(A+B)$ \\
\hline & & & Negative predictive value: & $D /(C+D)$ \\
\hline
\end{tabular}

\subsection{Analytical sensitivity}

(proportion of positive tests if the genotype is present)

The sensitivity of MLPA approaches $100 \%$ for deletion detection, but errors can be made when a polymorphism is present in the probe binding site, thus resulting in a false allele dropout as described by the manufacturer. The sensitivity for genomic sequencing also approaches $100 \%$ for mutation detection, but errors can be made because of polymorphisms causing allele dropout. Mutations outside the coding exons in promoters or enhancers are likely to be missed.

\subsection{Analytical specificity}

(proportion of negative tests if the genotype is not present) Analytical specificity is greater than $99 \%$. False positives in genomic sequencing are very rare as confirmation sequencing on an independent biological sample should be undertaken.

\subsection{Clinical sensitivity}

(proportion of positive tests if the disease is present)

The clinical sensitivity can be dependent on variable factors such as age or family history. In such cases a general statement should be given, even if quantification can only be made case by case.
The mutation detection rate for $S H O X$ or enhancer alterations or mutations is between 60 and $80 \%$ in LWD and between 2 and 5\% in ISS patients.

\subsection{Clinical specificity}

(proportion of negative tests if the disease is not present)

The clinical specificity can be dependent on variable factors such as age or family history. In such cases a general statement should be given, even if a quantification can only be made case by case.

$>99 \%$.

\subsection{Positive clinical predictive value}

(life time risk of developing the disease if the test is positive)

The penetrance of SHOX deficiency is high but its clinical expression is very variable, being more severe in females and becoming more pronounced with age.

\subsection{Negative clinical predictive value}

(Probability of not developing the disease if the test is negative) Assume an increased risk based on family history for a nonaffected person. Allelic and locus heterogeneity may need to be considered.

Index case in that family had been tested:

If a pathogenic SHOX mutation is identified in the index case, the negative predictive value is close to $100 \%$.

Index case in that family had not been tested:

Genetic heterogeneity with undiscovered genes or alternative regions of the PAR 1 regions means that $30 \%$ of LWD individuals who test negative for SHOX and the known enhancer regions may still have the condition.

\section{CLINICAL UTILITY}

3.1 (Differential) diagnosis: The tested person is clinically affected (To be answered if in 1.10 'A' was marked)

\subsubsection{Can a diagnosis be made other than through a genetic test?}

\begin{tabular}{lll}
\hline No & $\square$ (continue with 3.1.4) & \\
Yes & $\bigotimes$ & $\square$ \\
& Clinically & \\
& Imaging & \\
& Endoscopy & $\square$ \\
& Biochemistry & $\square$ \\
& Electrophysiology & $\square$ \\
& Other (please describe) & D Auxological data
\end{tabular}

A point system has been published but needs revision using our current knowledge of SHOX/PAR1 alterations. ${ }^{10}$ The more common clinical signs are shown in the table below:

\begin{tabular}{lll}
\hline $\begin{array}{l}\text { Clinical signs } \\
\text { (LWD) }\end{array}$ & $\begin{array}{l}\text { Clinical signs } \\
\text { of LMD }\end{array}$ & $\begin{array}{l}\text { Clinical signs } \\
\text { of ISS }\end{array}$ \\
\hline $\begin{array}{l}\text { Short stature (smaller than }-2 \text { SD), } \\
\text { although many individuals lie within } \\
\text { normal range }\end{array}$ & $\begin{array}{l}\text { Short stature } \\
(-6.0 \text { to }-9.0 \text { SD) }\end{array}$ & $\begin{array}{l}\text { Short stature } \\
\text { (smaller than } \\
\text { Mesomelic shortening of limbs }\end{array}$ \\
& $\begin{array}{l}\text { Mesomelic and } \\
\text { rhizomelic shortening } \\
\text { of limbs }\end{array}$ & \\
& $\begin{array}{l}\text { Madelung deformity } \\
\text { is rare }\end{array}$ &
\end{tabular}


Triangulation of the distal

epiphysis of the radius

Aplasia of the ulna

and fibula

Armspan:height ratio $<0.96$

Sitting height:height ratio

$<55.5$

Escoliosis (occasionally)

Severe hypoplasia of

the ulna and fibula

Cubitus Valgus (occasionally)
Thickened and curved

radius and tibia

\subsubsection{Describe the burden of alternative diagnostic methods to the patient}

Diagnosis can be established on the basis of appropriate clinical and $\mathrm{X}$-ray features, including mesomelic disproportion of the limbs and Madelung deformity of the forearm. However, SHOX deficiency causes short stature with a highly variable phenotype, depending upon the age and the sex of the patient. Genetic testing remains useful to confirm clinical diagnosis and to help prognosis evaluation.

3.1.3 How is the cost effectiveness of alternative diagnostic methods to be judged?

Although an accurate clinical assessment can aid the diagnosis of LWD or LMD and therefore establish the need for appropriate monitoring and management, genetic testing remains useful for genetic counselling and prenatal testing (LMD).

3.1.4 Will disease management be influenced by the result of a genetic test?

No $\square$

Yes $\otimes$

Therapy GH therapy has been approved by the FDA and EMEA

(please based on the data of a randomized controlled trial. ${ }^{11}$

describe)

Prognosis During the first year of therapy, the GH-treated cohort grew

(please faster than the untreated cohort and as fast as the girls with

describe) Turner syndrome, treated in parallel. The treated group gained $3.5 \mathrm{~cm}$ in the first year and $1.9 \mathrm{~cm}$ in the second year in comparison with the untreated group. ${ }^{11}$

Management Knowledge of the presence of SHOX mutations may

(please influence whether GH-treatment is suitable.

describe)
3.3.1 Does the result of a genetic test resolve the genetic situation in that family?

Yes.

3.3.2 Can a genetic test in the index patient save genetic or other tests in family members?

No. If a SHOX/PAR1 alteration is identified in the index patient, it can reduce the need for testing for other genetic conditions in family members by providing a diagnosis. However, LWD and LMD testing may still be required in other clinically affected family members.

3.3.3 Does a positive genetic test result in the index patient enable a predictive test in a family member?

Yes.

\subsection{Prenatal diagnosis}

3.4.1 Does a positive genetic test result in the index patient enable a prenatal diagnosis?

LWD is inherited in a pseudoautosomal dominant manner. Each child of an individual with LWD has a 50\% chance of inheriting the mutation. If both parents have LWD, the offspring have a $50 \%$ chance of having LWD, a $25 \%$ chance of having LMD, and a $25 \%$ chance of having neither condition., ${ }^{2,7}$ Prenatal testing is available but not common. It is mainly used in the possible outcome of LMD.

\section{IF APPLICABLE, FURTHER CONSEQUENCES OF TESTING}

Please assume that the result of a genetic test has no immediate medical consequences. Is there any evidence that a genetic test is nevertheless useful for the patient or his/her relatives? (Please describe)

The genetic diagnosis of LWD has clinical validity for both index cases and their relatives. Genetic counselling and appropriate predictive genetic testing of family members should establish their risk for the condition. Analysis of carrier status could be requested owing to the psychosocial implications of short stature and be useful for planned GH therapy.

\section{CONFLICT OF INTEREST}

The authors declare no conflict of interest.

\section{ACKNOWLEDGEMENTS}

This work was supported by EuroGentest2 (Unit 2: 'Genetic testing as part of health care'), a Coordination Action under FP7 (grant agreement number 261469) and the European Society of Human Genetics.
3.2 Predictive setting: The tested person is clinically unaffected but carries an increased risk based on family history

(To be answered if in 1.10 ' $\mathrm{B}$ ' was marked)

\subsubsection{Will the result of a genetic test influence lifestyle and prevention?}

If the test result is positive (please describe):

See above (item 3.1.4).

If the test result is negative (please describe):

No further follow-up is required.

3.2.2 Which options in view of lifestyle and prevention does a person at-risk have if no genetic test has been done (please describe)? Clinical follow-up should be undertaken.

3.3 Genetic risk assessment in family members of a diseased person (To be answered if in 1.10 ' $\mathrm{C}$ ' was marked)
1 Benito-Sanz S, Gorbenko Del Blanco D, Aza-Carmona M et al: PAR1 deletions downstream of SHOX are the most frequent defect in a Spanish cohort of Leri-Weill dyschondrosteosis (LWD) probands. Hum Mutat 2006; 27: 1062.

2 Huber C, Rosilio M, Munnich A, Cormier-Daire V, French SHOX GeNeSIS Module: High incidence of SHOX anomalies in individuals with short stature. J Med Genet 2006; 43 : 735-739.

3 Chen J, Wildhardt G, Zhong Z et al: Enhancer deletions of the SHOX gene as a frequent cause of short stature: the essential role of a $250 \mathrm{~kb}$ downstream regulatory domain. J Med Genet 2009; 46: 834-839.

4 Benito-Sanz S, Aza-Carmona M, Rodríguez-Estevez A et al: Identification of the first PAR1 deletion encompassing upstream SHOX enhancers in a family with idiopathic short stature. Eur J Hum Genet 2011; 19: 1218-1225.

5 Benito-Sanz S, Royo JL, Barroso $E$ et al: Identification of the first frequent PAR1 deletion in Léri-Weill dyschondrosteosis and idiopathic short stature reveals the presence of a novel SHOX enhancer. J Med Genet 2011 (in revision).

6 Schneider KU, Marchini A, Sabherwal N et al: Alteration of DNA binding, dimerization, and nuclear translocation of SHOX homeodomain mutations identified in idiopathic short stature and Leri-Weill dyschondrosteosis. Hum Mutat 2005; 26: 44-52.

7 Niesler B, Röth R, Wilke S, Fujimura F, Fischer C, Rappold G: The novel human SHOX allelic variant database. Hum Mutat 2007; 28: 933-938. 
8 Barca-Tierno V, Aza-Carmona M, Barroso E et al: Identification of a Gypsy SHOX mutation (p.A170P) in Léri-Weill dyschondrosteosis and Langer mesomelic dysplasia. Eur J Hum Genet 2011; 19: 1218-25.

9 Benito-Sanz S, Barroso E, Heine-Suñer D et al: Clinical and molecula evaluation of SHOXIPAR1 duplications in Leri-Weill dyschondrosteosis (LWD) and idiopathic short stature (ISS). J Clin Endocrinol Metab 2011; 96 E404-E412.
10 Rappold G, Blum WF, Shavrikova EP et al: Genotypes and phenotypes in children with short stature: clinical indicators of SHOX haploinsufficiency. J Med Genet 2007 44: 306-313.

11 Blum WF, Crowe BJ, Quigley CA et al, SHOX Study Group: Growth hormone is effective in treatment of short stature associated with short stature homeobox-containing gene deficiency: Two-year results of a randomized, controlled, multicenter trial. J Clin Endocrinol Metab 2007; 92: 219-228. 